\title{
Gestión de mantenimiento en pymes industriales*
}

\author{
Ortiz Useche, Alexis** \\ Rodríguez Monroy, Carlos*** \\ Izquierdo, Henry****
}

\section{Resumen}

En este artículo se realiza un análisis de la gestión de mantenimiento en las pymes industriales de Ciudad Guayana, Estado Bolívar. Para caracterizar estas empresas, se aplicó un cuestionario a gerentes, jefes y supervisores de mantenimiento pertenecientes a una muestra de setenta y cinco (75) pymes del sector industrial, de una población de doscientas (200) empresas. A través de la aplicación del análisis factorial exploratorio, se estructuró un modelo de gestión de mantenimiento basado en el ciclo Planear, Hacer, Verificar y Actuar (PHVA). La calificación obtenida para el conjunto de empresas evaluadas ( $57 \%$ de cumplimiento), pone de relieve la debilidad de la gestión de mantenimiento en las pymes industriales, particularmente en los aspectos de planificación y mejora continua; la mayoría de las pymes evaluadas se encuentran en la etapa de mantenimiento correctivo, siendo su norma de actuación la respuesta solo a la ocurrencia de averías. El reto planteado a las pymes de la región es evolucionar del paradigma de la corrección a la práctica de la prevención, para lo cual el empresario debe concebir al mantenimiento como un elemento de competitividad en lugar de un mal necesario.

Palabras clave: Gestión del mantenimiento, planificación del mantenimiento, ejecución, verificación, mejora.

Recibido: 27-09-11. Aceptado: 27-02-13

* Este trabajo constituye un avance del Proyecto de Investigación "Modelo de evaluación y mejora de la gestión empresarial. Aplicación a las Pymes de Ciudad Guayana", adscrito al Centro de Investigaciones Gerenciales de Guayana (CIGEG) de la UNEG.

** Investigador-docente de la UNEG, adscrito al CIGEG. Cursante del Doctorado en Administración de Empresas de la Universidad Politécnica de Madrid. Ingeniero industrial, Especialista en Operaciones y Producción, Magister en Gerencia, e-mail: aortiz@uneg.edu.ve

*** Investigador-docente de la UPM, Doctor adscrito al Departamento de Ingeniería de Organización, Administración de Empresas y Estadística. Escuela Técnica Superior de Ingeniería Industrial. Universidad Politécnica de Madrid, España, e-mail: crmonroy@etsii.upm.es.

**** Investigador-docente de la UNEG, Coordinador del Centro de Investigaciones Gerenciales de Guayana. Doctor en Administración de Empresas y Estadística por la Universidad Politécnica de Madrid, e-mail: hizquier@uneg.edu.ve 


\title{
Maintenance Management in Industrial SMEs
}

\begin{abstract}
This article analyzes the management of maintenance for industrial SMEs in the City of Guayana, State of Bolivar. To characterize these companies, a questionnaire was applied to maintenance managers, chiefs and supervisors belonging to a sample of seventy-five (75) small and medium enterprises in the industrial sector, out of a population of two-hundred (200) companies. By applying exploratory factor analysis, a model for managing maintenance was structured, based on the cycle Plan, Do, Check and Act (PDCA). The rating obtained for the set of companies evaluated was $57 \%$ fulfillment, placing in relief the weakness of maintenance management in industrial SMEs, particularly regarding aspects of planning and continual improvement. Most of the evaluated SMEs are in the corrective maintenance stage, and their norm for action is merely responding when breakdowns occur. The challenge posed to SMEs of the region is to evolve from the correction paradigm to practicing prevention; to accomplish this, the impresario should conceive maintenance as an element of competitiveness instead of a necessary evil.
\end{abstract}

Keywords: Maintenance management, maintenance planning, execution, checking, improvement.

\section{Introducción}

La creciente importancia de las pymes en los procesos de desarrollo económico de los países latinoamericanos se debe, de acuerdo con estadísticas del Sistema Económico Latinoamericano y del Caribe (SELA, 2009:9), a su contribución al empleo (alrededor del 35-40\% de la población económicamente activa), su aporte al PIB regional (alrededor del $33 \%$ ) y en promedio el $25 \%$ de las exportaciones (Zevallos, 2008:32-33).

Hoy en día, estas empresas deberían poder desarrollar todas sus capacidades de adaptación a fin de competir en mercados cada vez más extensos, dinámicos y exigentes; en una época signada por la globalización de los mercados y la crisis económica mundial.

Según Martínez et al. (2009:28,94), entre los factores determinantes en la mejora de la posición competitiva de la empresa se encuentran la innovación tecnológica, la internacionalización, la financiación, la gestión de los recursos humanos y el desarrollo de prácticas de gestión, dirigidas a mejorar la eficiencia y productividad de los procesos productivos, para tratar de alcanzar la mayor rentabilidad de la inversión realizada en los activos industriales.

Sin embargo, la presencia de fallas y averías en las instalaciones industriales trae consigo un aumento en los costos de operación y pérdida de ingresos, por lo que es indiscutible que la gestión de mantenimiento cobre mayor relevancia debido al impacto directo que tiene sobre el proceso productivo.

El mantenimiento ha sido considerado como un proceso de apoyo a la producción, y como tal, consumidor voraz de recursos, al que se ha tendido a minimizar y en los últimos años a subcontratar (práctica recientemente prohibida en Venezuela por la Ley Orgánica del Trabajo), de modo que las pymes deben asumir di- 
rectamente la gestión de mantenimiento; no obstante, en la medida que a los procesos productivos se les ha exigido mayor eficacia y calidad, la necesidad de optimizar la función mantenimiento ha hecho que se valore el impacto real de la misma desde el punto de vista del valor que puede aportar para la empresa.

Las consideraciones anteriores dan origen a esta investigación, que tiene dos objetivos: realizar un análisis de la gestión de mantenimiento en las pymes industriales de Ciudad Guayana y diseñar un modelo de evaluación y mejora de dicha gestión.

Este modelo proporcionará a las pymes de la región una herramienta adaptada a las características y especificidades propias de este tipo de empresas, a la vez le permitirá evaluar exhaustivamente sus prácticas en mantenimiento e identificar áreas y acciones de mejora para incrementar la eficacia de la gestión.

Para analizar la gestión de las pymes industriales, se elaboró un cuestionario, diseñado de acuerdo con la técnica de escalamiento tipo Likert; una vez validado el cuestionario, se aplicó a un grupo de setenta y cinco (75) pymes del sector industrial, ubicadas en el Estado Bolívar, Venezuela.

Para construir el modelo, se utilizó la técnica del análisis factorial exploratorio con rotación varimax, por medio del software estadístico SPSS. Los factores determinados se agruparon de acuerdo al ciclo Planear, Hacer, Verificar, Actuar (PHVA).

Desde el punto de vista empresarial, esta investigación brinda a las pymes información valiosa sobre el estado actual de su gestión de mantenimiento así como herramientas, para organizar y me- jorar la misma, el modelo propuesto puede ser usado como una herramienta; desde el punto de vista académico, pues proporciona una guía a los estudiantes de Mantenimiento Industrial para comprender mejor la interrelación de las variables envueltas en este proceso.

\section{Gestión de mantenimiento: Consideraciones teóricas}

Según Oliva et al. (2010:131), el mantenimiento es un servicio que agrupa una serie de actividades mediante las cuales un equipo, máquina, construcción civil o instalación, se mantiene o se restablece a un estado apto para realizar sus funciones, siendo importante en la calidad de los productos y como estrategia para una competencia exitosa.

El objetivo básico de cualquier gestión de mantenimiento, consiste en incrementar la disponibilidad de los activos, a bajos costos, permitiendo que dichos activos funcionen de forma eficiente y confiable dentro de un contexto operacional (Amendola, 2006: 45).

Existen diferentes metodologías para abordar la gestión de mantenimiento, entre ella destacan el mantenimiento centrado en la confiabilidad, RCM por sus siglas en inglés (Reliability Centered Maintenance o Mantenimiento Centrado en la Confiabilidad) y el mantenimiento productivo total, TPM por sus siglas en inglés (Total Productive Maintenance o Mantenimiento Productivo Total).

El RCM es un proceso usado para determinar qué es lo que debe hacerse para asegurar que cualquier activo físico se mantenga operando de acuerdo a las necesidades del usuario, en el contexto 
operacional presente (Kister y Hawkins, 2006:98).

Según Cuatrecasas y Torrell (2010: 36), el Mantenimiento Productivo Total (TPM) es una filosofía preventiva desde el diseño, pasando por la mejora hasta la prevención de problemas, que tiene como objetivos eliminar las seis grandes pérdidas debidas a 1) paradas/averías, 2) cambios de útiles/ajustes, 3) tiempo no usado y paradas breves, 4) pérdidas de velocidad, 5) defectos y, 6) pérdidas debidas a puesta en marcha; que permitan mejorar rendimientos utilizando medios como el
Mantenimiento Autónomo, el Mantenimiento Planificado y la Prevención de Mantenimiento.

Por otra parte el ciclo PHVA es considerado como una metodología para la mejora continua de la gestión. Fue desarrollada por Walter Shewhart y nombrada como el ciclo Deming por los japoneses en 1950.

En este estudio se asumen las siguientes consideraciones acerca de las actividades del ciclo PHVA, las cuales constituyen la guía o referencia para identificar las variables que constituyen la gestión de mantenimiento (Cuadro 1).

\section{Cuadro 1 \\ Actividades del ciclo PHVA}

\begin{tabular}{|c|c|}
\hline Ciclo (PHVA) & Actividades \\
\hline Planear & $\begin{array}{l}\text { - Actividades dirigidas a determinar los requerimientos especificados por el cliente, } \\
\text { requerimientos no especificados por el mismo pero necesarios para la prestación } \\
\text { del servicio y requisitos legales o regulatorios relacionados con servicio. } \\
\text { - Definición de objetivos y preparación de cursos de acción para alcanzarlos. } \\
\text { - Identificación de estándares aplicables; desarrollo de estándares. } \\
\text { - Definición de medios y recursos a ser utilizados y los términos de su empleo. } \\
\text { - Establecimiento de procedimientos, estructuras y tiempos para el desarrollo de } \\
\text { las actividades previstas. } \\
\text { - Determinación de instrumentos y modalidades de coordinación y de control. } \\
\text { - Asignación de responsabilidades operativas y de control. } \\
\text { - Desarrollo de programas operativos detallados; programación del trabajo. } \\
\text { - Elaboración de instrucciones para llevar a cabo los trabajos, determinación de } \\
\text { necesidades de información para el control del progreso, asignación individual } \\
\text { de tareas y trabajo compartido entre individuos. } \\
\text { - Diseño de procesos tales como verificación, validación, monitoreo e inspección. } \\
\text { - Preparación de documentación. }\end{array}$ \\
\hline Hacer & $\begin{array}{l}\text { - Implementación; ejecución de las actividades planeadas; cubre las diferentes } \\
\text { actividades que la empresa necesita ejecutar durante la gestión. }\end{array}$ \\
\hline Verificar & $\begin{array}{l}\text { - Actividades que se realizan para comparar los resultados de la ejecución con } \\
\text { los objetivos para determinar que los mismos se han alcanzado; cubre las acti- } \\
\text { vidades de medición, verificación, validación, monitoreo e inspección de los } \\
\text { procesos; análisis de datos. }\end{array}$ \\
\hline Actuar & - Actividades dirigidas al mejoramiento continuo; acciones preventivas y correctivas. \\
\hline
\end{tabular}

Fuente: Adaptado de ISO (2008:8); Gil y Giner (2010:199-203). 
Gestión de mantenimiento en pymes industriales

Ortiz Useche, Alexis; Rodríguez Monroy, Carlos e Izquierdo, Henry

Las actividades contenidas en el cuadro anterior, son descritas desde una perspectiva teórica, enmarcadas en cada uno de los procesos característicos de la gestión de mantenimiento, como sigue:

\subsection{Planificación del mantenimiento}

De acuerdo con Stephens (2010: 7), la planificación del mantenimiento, incluye la definición de políticas y objetivos. Se definen objetivos técnico-económicos del servicio así como los métodos a implantar y los medios necesarios para alcanzarlos. Los objetivos son los resultados específicos que pretende alcanzar la organización de mantenimiento a mediano y largo plazo; deben incluir un plazo de ejecución, poseer indicadores, metas y responsables, de tal manera que puedan ser evaluados.

Por otra parte, García (2003:7-14), plantea la identificación y clasificación de los equipos; actividad que consiste en disponer de un inventario donde estén claramente identificados y clasificados. Se recomienda un sistema tipo árbol y un código que identifique el departamento y la unidad, además de los códigos específicos del equipo. Debe registrarse también: fecha de instalación, estado o condición, información técnica, historia del equipo (incluye fallas registradas y tipo de fallas), herramientas especiales y procedimientos de seguridad. Otra información relevante para mantenimiento, la constituye las condiciones de trabajo, y de diseño, recomendaciones del fabricante y los aspectos legales.

Con respecto al análisis de criticidad de equipos, ésta es una metodología que permite establecer la jerarquía o prioridades de procesos, sistemas y equipos, creando una estructura que facilita la toma de decisiones acertadas y efectivas, dirigiendo los esfuerzos y recursos a áreas donde sea más importante y/o necesario mejorar la confiabilidad operacional, basado en la realidad actual (Amendola, 2006:31).

Al respecto, García (2003:25) propone tres niveles de importancia o criticidad: 1) equipos críticos; 2) equipos importantes y 3) equipos prescindibles. Para clasificar cada uno ellos, debe considerarse la influencia que una falla tendría en aspectos como: producción, calidad, mantenimiento y seguridad.

Para la selección del tipo de mantenimiento a aplicar: en el RCM se distinguen los siguientes:

- Mantenimiento predictivo o a condición: consiste en la búsqueda de indicios o síntomas que permitan identificar una falla antes de que ocurra.

- Mantenimiento preventivo: referido a tareas de sustitución o retrabajo hechas a intervalos fijos independientemente del estado del elemento o componente.

- Mantenimiento correctivo: consiste en reparar el equipo una vez que la falla ocurre; no se lleva a cabo ninguna tarea proactiva (predictiva o preventiva) para manejar la falla.

- Mantenimiento de detección o búsqueda de fallas: consistente en la prueba de dispositivos de protección bajo condiciones controladas, para asegurarse que estos dispositivos sean capaces de brindar la protección requerida cuando sean necesarios. 
Por otra parte en lo que respecta a la metodología Failure Modes and Effects Analysis (FMEA), conocida como Análisis del Modo y Efectos de Fallo (AMEF), Goble (2010:87) lo define como una técnica sistemática diseñada para identificar problemas o para el análisis de modos de fallo y efectos. La implementación de esta metodología comienza con una lista detallada de todos los componentes pertenecientes al sistema que se analiza. El sistema completo puede analizar un componente a la vez por medio de una estructura jerárquica. Para cada componente, se identifican todos los modos de falla conocidos; y para cada componente/modo de fallo se hace una lista de los efectos en el siguiente nivel. Finalmente, para cada componente/modo de fallo se lista la severidad del efecto y posteriormente se determinan las medidas preventivas que eviten o amortigüen los efectos de los fallos.

Así, la planificación del mantenimiento, consiste en seleccionar las tareas de mantenimiento que se ajustan al modelo de mantenimiento determinado para cada sistema. La planificación de mantenimiento debe estar ligada a la planificación y programación de la producción, partiendo según González (2005: 393-394), de una planificación a largo plazo; para con esta base elaborar el presupuesto anual en el que se incorporan tanto los recursos humanos necesarios, como los materiales, los suministros y los servicios externos.

Con respecto a la estimación de los tiempos de ejecución del mantenimiento, la razón principal es utilizar las estimaciones de tiempos de actividades como variable de entrada a la programación del mantenimiento y nivelar los recursos re- queridos para estas actividades. La estimación puede hacerse considerando la historia técnica y la experiencia, aunque es recomendable efectuar la estructura de partición del trabajo de mantenimiento al respecto.

Cabello (2002:117), establece que la empresa debe contar con un programa mensual que incluya los programas de lanzamiento de rutas de actividades con sus órdenes detalladas y, finalmente se programa detalladamente la ejecución del mantenimiento de manera semanal y diaria. Esto lo denomina programación del mantenimiento y define como el conjunto de acciones a las que se somete un sistema, para que conserve y/o controle a todos sus subsistemas y componentes, con las condiciones de funcionamiento previamente especificadas para su ciclo de vida. Una vez elaborada la lista de tareas que componen el plan de mantenimiento es conveniente agruparlas a fin de facilitar su ejecución. Esta agrupación se conoce como rutas o gamas de mantenimiento (García, 2003:79). Dichas rutas pueden ser diarias contentivas de tareas como controles visuales, mediciones y pequeños trabajos de limpieza y/o engrase; las rutas semanales y mensuales comprenden tareas más complicadas como desmontajes, paradas de equipos y engrases; las rutas o gamas anuales comprenden por lo general una revisión completa del equipo (over haul) o grandes reparaciones y suponen la parada del equipo por varios días; deben estar previstas en el presupuesto anual.

Para gestionar adecuadamente las órdenes de trabajo, es necesaria la priorización de actividades que se generan. De acuerdo con García (2003:103): 
- Averías urgentes: deben resolverse inmediatamente.

- Averías importantes: aunque causan trastornos pueden esperar a que todas las averías urgentes estén resueltas.

- Averías cuya solución puede programarse: son averías que causan pequeños trastornos, por lo que se podría esperar a una parada del equipo para resolverlos.

Por otra parte, en toda gestión de mantenimiento debe definirse la política de repuestos que soporte dicha gestión (considerando tanto las tareas planificadas como las no planificadas). Wenyuan (2005), plantea que a partir de las tareas de mantenimiento se debe identificar el conjunto de repuestos necesarios para cumplir dichas tareas. Cada repuesto debe tener un código y una breve descripción, una vez identificados, se deben determinar los niveles adecuados de repuestos, considerando un balance entre el "costo" de tener el repuesto, y el "costo" de no tenerlo (costo de oportunidad) incluyendo no sólo el riesgo económico sino también el riesgo sobre la seguridad, el medio ambiente, la calidad de servicio, entre otros.

De acuerdo con Zylberberg (2006: 4-5), para cada repuesto (o grupo de repuestos) debe determinarse cuál es el criterio u objetivo en base al cual la decisión respecto a la política de inventarios debe ser tomada, es decir, de que formas puede importar tener o no tener un repuesto; una vez determinado el objetivo de la política de inventarios para el repuesto que se está analizando (maximizar disponibilidad, minimizar tasa de faltantes, entre otros), se debe traducir este objetivo en una política de repuestos concreta: ¿se necesita el repuesto? Y si es así, ¿cuánto se necesita? En esta etapa debe analizarse si puede cumplirse con el objetivo propuesto sin necesidad de mantener repuestos en almacén, y en caso de que la respuesta a esta pregunta sea negativa debe determinarse qué nivel de inventario es requerido.

La organización del mantenimiento, según la norma venezolana COVENIN 2500-93 (1993:4) debe estar dimensionada con respecto al tamaño del sistema productivo, tipo de equipos a mantener, tipo de proceso y distribución geográfica, de tal manera que el personal sea suficiente tanto en cantidad como en calificación y su rendimiento sea acorde con los objetivos establecidos; de acuerdo con la norma, la estructura organizativa debe estar definida con su respectivo organigrama actualizado y conservarse por escrito las diferentes funciones y responsabilidades para los distintos componentes dentro de la organización del mantenimiento.

De igual forma debe existir una documentación del mantenimiento, formada por documentación técnica y documentación relativa a la ejecución del mantenimiento propiamente dicho; esta documentación debe estar integrada al sistema general de la organización.

La norma AENOR UNE-EN 13269 (2007:8), establece que la documentación técnica comprende: datos técnicos, manuales de operación, manual de mantenimiento, lista de componentes (organización y detalle), mapa de lubricación, diagramas (unifilar, lógico, circuitos, tuberías, instrumentos y elementos de medida y control), localización, layout, programa de pruebas y certificados, los cua- 
les deben ser proporcionados por los fabricantes. La norma señala también que la documentación relativa a la ejecución del mantenimiento comprende: protocolos de mantenimiento, listas de revisión, procedimientos, instrucciones de trabajo, formularios y fichas de control.

Un elemento esencial de la documentación de mantenimiento es la orden de trabajo, según García (2003:250), es el documento en el que la gerencia de mantenimiento informa al operario o al técnico de mantenimiento sobre la tarea que tiene que realizar. Estas órdenes son una de las fuentes de información más importantes de mantenimiento, pues en ella se recogen los datos más importantes de cada intervención.

Por último, la planificación de actividades deberá partir de una planificación a largo plazo; con esta base se elabora el presupuesto anual en el que se incorporan tanto los recursos humanos necesarios, como los materiales, los suministros y los servicios externos (González, 2005: 393-394).

\subsection{Ejecución del mantenimiento (hacer)}

Para García (2003:43), la ejecución del plan de mantenimiento comprende inspecciones visuales, lubricación, verificaciones de funcionamiento, limpiezas técnicas condicionales, y sistemáticas, ajustes condicionales y sistemáticos, sustitución sistemática de piezas y sustitución de piezas sometidas a desgaste. Asimismo, se requiere de reparaciones y correcciones; $y$, aunque lo deseable es que todas las actividades de mantenimiento sean planificadas, siempre existe una pequeña probabilidad de fallas imprevistas, con la necesidad de aplicar.

\subsection{Verificación del mantenimiento}

Para verificar que la ejecución se esté realizando de manera correcta, se realizan análisis de averías, cuyo objetivo es determinar las causas que las provocan, para adoptar medidas preventivas que las eviten (García, 2003:111). Cuando un equipo o una instalación falla, lo hace generalmente por uno de los siguientes motivos: por un fallo en el material, por un error humano del personal de operación, por un error humano del personal de mantenimiento y por condiciones externas anómalas. En ocasiones, confluyen en una avería más de una de estas causas.

La evaluación de la efectividad del mantenimiento, puede medirse a través de indicadores; éstos deben estar relacionados con la gestión del mantenimiento; básicamente se asocian con: eficiencia y productividad, organización y recursos humanos, servicios externos, costos, calidad y percepción del cliente y resultados técnicos (González, 2004:39).

Sobre el particular Stephens (2010: 30), Plaza (2009:132) y Arata (2009: 134), proponen los siguientes:

- Costo anual de mantenimiento: parámetro importante en la elaboración de presupuestos anuales de costos de operación y mantenimiento de sistemas.

- Tiempo medio entre reparaciones: indica la frecuencia esperada de tareas de mantenimiento correctivo (frecuencia de averías o fallas). 
- Tiempo Promedio entre Fallas (MTBF): valor esperado o medio del tiempo para la variable aleatoria de fallo. Este indicador mide el tiempo promedio que es capaz de operar el equipo a capacidad sin interrupciones dentro del período considerado y es una medida de la fiabilidad.

- El Tiempo Promedio de Reparación (MTTR): es la medida de la distribución del tiempo de reparación de un equipo o sistema. EI MTTR mide la efectividad en restituir la unidad a condiciones óptimas de operación una vez que la unidad se encuentra fuera de servicio por una falla, dentro de un período de tiempo determinado, y considerando al tiempo de fallo igual al tiempo para reparar. Es una medida de la mantenibilidad.

- Disponibilidad (D): se define como la capacidad del equipo o instalación para realizar una función requerida bajo condiciones específicas sobre un período de tiempo determinado, asumiendo que los recursos externos requeridos son suministrados.

En otro orden de ideas, la organización de mantenimiento debe realizar el seguimiento de la información relativa a su gestión. Debe considerar el cumplimiento de los requisitos de los clientes; siendo las fuentes de información principales el cliente; a quien se aplican cuestionarios y encuestas, midiendo con ello su satisfacción.

Por último, las mejoras y/o modificaciones de las instalaciones, según García (2003:46), pueden estar asociadas a cambios en: materiales, diseño de una pieza, instalación de sistemas de detección, diseño de una instalación y, condi- ciones externas a la pieza o equipo; la modificación de procedimientos de operación y mantenimiento tiene como objetivo minimizar la incidencia del personal de operación en los problemas del equipo y reducir las averías por intervenciones incorrectas del personal de mantenimiento.

\subsection{Mejoramiento continuo (actuar)}

En este proceso se encuentran la mantenibilidad, definida por Creus (2005:95) como el conjunto de factores o elementos de que se dispone para realizar el mantenimiento de un sistema, es decir, es una medida de la facilidad con que el sistema o equipo puede mantenerse. Para Arata (2009:131), la mantenibilidad de un equipo está condicionada a los tiempos referidos a: preparación, localización de la falla, desmontaje, obtención de la piezas y materiales necesarios, reparación propiamente ajuste y calibración, montaje, comprobación del buen funcionamiento del componente reparado y limpieza; lo cual implica que una reducción en cualquiera de estos tiempos llevará a una mejora de la mantenibilidad.

El mantenimiento puede ser autónomo, definido como una técnica para conseguir que los operarios se involucren en el cuidado de los equipos, trabajando con mantenimiento para estabilizar las condiciones de los mismos; comprende la ejecución de rutinas de inspección y limpieza, lubricación, ajustes y reparaciones menores (Campbell et al., 2010:219). De acuerdo con González (2005:109) el mantenimiento autónomo se basa en los principios de las $5 \mathrm{~S}$ cuyo significado es el siguiente: 
- Organización (Seiri): en el puesto de trabajo no debe haber más que el herramental necesario para la operación o producción de dicho puesto y su mantenimiento. Dichas herramientas de producción deben estar adecuadamente organizadas, codificadas y en el lugar preciso.

- Orden (Seiton): los elementos, repuestos y utillajes deben estar ordenados en ubicaciones específicas.

- Limpieza e inspección (Seiso): aprovechamiento de las operaciones de limpieza que deben realizar los operarios de producción para llevar a cabo las inspecciones.

- Estandarización o normalización (Seiketsu): empleo de estándares, etiquetas, colores, entre otros, como herramientas facilitadoras para el mantenimiento autónomo.

- Cumplimiento o disciplina (Shitsuke): las rutinas de limpieza e inspección que se definan conjuntamente con producción, así como el mantenimiento del orden y limpieza, son básicos para que el área de trabajo sea conforme con los estándares de auto mantenimiento.

\section{Gestión de mantenimiento en las Pymes de Ciudad Guayana}

Para caracterizar las pymes industriales, se elaboró un cuestionario con las variables identificadas en la revisión del estado del arte, realizada sobre la gestión de mantenimiento, la validez del cuestionario se determinó mediante la técnica del juicio de expertos; en cuanto a la con- fiabilidad, se realizó una prueba piloto en diez empresas de la población, el Alfa de Cronbach fue de 0,93 , por lo que se considera que el instrumento tiene confiabilidad aceptable (Alpha $\geq 0,80$ ).

Una vez validado el cuestionario, se aplicó el mismo a un grupo de pymes del sector industrial, ubicadas en el Estado Bolívar, Venezuela, específicamente en Ciudad Guayana. En este estudio se considera como población las pymes industriales de Guayana inscritas en las Cámaras Industriales de la región, aproximadamente doscientas empresas (200). De este número, solo accedieron a someterse al estudio setenta y cinco (75) empresas, es decir, el $38 \%$. En total, se llevaron a cabo trescientas (300) entrevistas a gerentes, jefes y supervisores de mantenimiento, en un trabajo de campo realizado en el año 2011. El error muestral del estudio es de $\pm 9 \%$, con un nivel de confianza de $95 \%(z=1,96)$ y el tratamiento de la información se llevó a cabo utilizando el software SPSS versión 15.

El procedimiento utilizado para realizar el análisis factorial exploratorio fue:

a) Cálculo e interpretación de la matriz de correlaciones para las variables consideradas. Para ello, se recurrió a los siguientes indicadores:

- Coeficientes de correlación de Pearson: a mayor valor de los coeficientes de correlación mayor será la relación entre las variables. Si la correlación lineal entre las variables es nula, la técnica no es aplicable. Los valores resultantes en la matriz de correlaciones están entre $-0,184$ y 0,825 , lo que demuestra que existe una elevada relación entre las variables. 
- Contraste de esfericidad de Barlett: si el nivel crítico (p-valor o sig.) es 0,000, existe correlación significativa entre las variables. Si el nivel crítico ( $p$-valor o sig.) > 0,05 no existen garantías de que el modelo factorial sea el más adecuado para explicar los datos. Para los datos evaluados se obtuvo un p-valor $=0,000$ por lo que se deduce que existe correlación significativa.

- Índice Kaiser, Meyer y Olkin (KMO): si $\mathrm{KMO}>0,5$ los datos se adecúan a un modelo factorial. Si KMO $<0,5$ no hay adecuación. La medida de adecuación muestral resultante fue 0,852 , por lo que se considera aceptable el uso del análisis factorial.

- Determinante de la matriz de correlaciones: si este valor es muy pequeño, señala que las variables estudiadas están linealmente relacionadas, y existe la opción de reducir la dimensión. Su resultado es $1,66.10^{-7}$, lo cual señala que el grado de correlación entre las variables es muy alto.

b) Extracción de factores: se utilizó el método de componentes principales para transformar un conjunto de variables interrelacionadas en un nuevo conjunto de variables, combinación lineal de las primeras. Se utilizaron los factores cuyos auto valores iniciales son mayores que 1 , de acuerdo con los valores presentados en la matriz de correlaciones.

c) Rotación de factores: se aplicó la rotación ortogonal Varimax. Con esta técnica se logra que los pesos de las variables en los factores tengan la mayor varianza posible; esto significa que los ítems tienden a tener correlaciones altas con unos factores y muy bajas en otros; por esto es la solución más clara para diferenciar conjuntos de ítems.

d) Interpretación de factores: en primer lugar, se identifican las variables cuyas correlaciones con el factor son las más elevadas en valor absoluto (se eliminan las cargas factoriales menores que $0,5)$ y segundo, se otorga un nombre a los factores, de acuerdo con la estructura de sus correlaciones con las variables.

La aplicación de la técnica estadística agrupó veinte (20) variables en cuatro factores con una varianza total explicada de 68,510\%; según Salvador y Gargallo (2006), se debe dar un nombre a estos factores, el cual se asigna de acuerdo con la estructura de sus correlaciones con las variables. De esta manera, los factores se han denominado Planificar, Hacer, Verificar y Actuar. El primer factor, que representa el 41,366\% de la varianza, está compuesto por doce (12) variables que se agruparon en un bloque denominado Planificar, debido a la naturaleza de estas variables; el segundo factor $(12,944 \%$ de la varianza) involucra la variable ejecución del plan de mantenimiento y la variable reparación y corrección, agrupadas como Hacer; el tercer factor $(8,168 \%$ de la varianza) está constituido por Cuatro (4) variables, que constituyen el bloque Verificar; el cuarto factor $(6,032 \%$ de la varianza) incluye las variables mejora de la mantenibilidad y mantenimiento autónomo $y$ $5 S$, y se ha denominado Actuar (Mejora Continua).

En la matriz de la Tabla 1 se aprecia la estructura de los componentes rotados, y se muestra además la distribución de las variables por factor. 
Tabla 1

Matriz de componentes rotados

\begin{tabular}{|c|c|c|c|c|c|}
\hline \multirow[b]{2}{*}{ Factores } & \multirow[b]{2}{*}{ Variables } & \multicolumn{4}{|c|}{ Componente } \\
\hline & & 1 & 2 & 3 & 4 \\
\hline \multirow[t]{12}{*}{ Planificar } & Políticas y objetivos & ,861 & & & \\
\hline & Organizar mantenimiento & ,876 & & & \\
\hline & Definir documentación & ,781 & & & \\
\hline & Identificar y clasificar equipos & ,778 & & & \\
\hline & Establecer presupuesto & ,627 & & & \\
\hline & Analizar criticidad equipos & ,782 & & & \\
\hline & Seleccionar modelo mantto. & ,706 & & & \\
\hline & Análisis AMEF &, 554 & & & \\
\hline & Planificar mantenimiento & ,872 & & & \\
\hline & Estimar tiempos ejecución & ,657 & & & \\
\hline & Establecer política repuestos & ,614 & & & \\
\hline & Programar mantenimiento &, 736 & & & \\
\hline \multirow{2}{*}{$\begin{array}{l}\text { Hacer } \\
\text { (ejecutar) }\end{array}$} & Ejecutar plan mantenimiento & & & ,758 & \\
\hline & Reparar y corregir & & & 914 & \\
\hline \multirow[t]{4}{*}{ Verificar } & Analizar averias & & ,785 & & \\
\hline & Evaluar efectividad mantenimiento & & ,913 & & \\
\hline & Mejorar modificar instalaciones & & ,795 & & \\
\hline & Medir satisfacción del cliente & & ,703 & & \\
\hline \multirow{2}{*}{$\begin{array}{c}\text { Actuar } \\
\text { (mejora } \\
\text { continua) }\end{array}$} & Mejorar mantenibilidad & & & & ,733 \\
\hline & Mantenimiento autónomo y $5 \mathrm{~S}$ & & & & ,806 \\
\hline
\end{tabular}

Fuente: Elaboración propia.

Los resultados obtenidos del cuestionario aplicado al sector industrial de pymes se han utilizado para caracterizar la gestión de mantenimiento y determinar tanto los aspectos de mayor fortaleza, que deben ser sostenidos, como los de mayor debilidad, que deben ser mejorados. El instrumento de evaluación fue diseñado para asignar puntos de acuerdo al grado de cumplimiento de cada variable; las variables con baja puntuación re- presentan áreas de mejora en la gestión. Así, como resultado de la evaluación, el conjunto de empresas obtuvo un valor en cumplimiento porcentual, indicativo de su nivel de gestión del mantenimiento. No obstante, la puntuación es anecdótica y sirve solo para priorizar actuaciones y poder comparar resultados.

La evidencia empírica recolectada se estructuró de acuerdo a los cuatro procesos fundamentales del modelo: Planifi- 
car, Hacer (ejecutar), Verificar y Actuar (Mejora continua). El resultado global de cumplimiento para las setenta y cinco empresas fue de $57 \%$. A continuación, se muestran en detalle los resultados obtenidos para cada una de las variables.

\subsection{Planificación}

En función de los resultados obtenidos (Tabla 2), se evidencia que muy pocas empresas han definido una política de mantenimiento, aunque la mayoría ha establecido objetivos. Los equipos se encuentran identificados y clasificados, con sistemas de codificación no significativos (el código no aporta ninguna información adicional).

No se lleva un análisis formal de criticidad de equipos, se conocen cuales son los equipos críticos, pero no se ha cuantificado la incidencia de la falla de un equipo sobre otros; el modelo de mantenimiento mayormente usado es el correctivo (comprende inspecciones visuales, lubricación y reparación de las fallas que surjan), aunque algunas empresas están tratando de consolidar el mantenimiento preventivo (del predictivo no se tiene conocimiento); se elabora un plan general de mantenimiento que después se convierte en un programa, con un somero análisis previo de los modos de fallo y efectos y una débil estimación de los tiempos de ejecución, lo que dificulta estimar el volumen de trabajo de mantenimiento que se puede ejecutar; no se contempla en el plan una revisión completa de los equipos (over haul); se repara cuando el equipo falla pero la falta de definición de prioridades impide la gestión adecuada de las órdenes de trabajo.
Tabla 2

Planificación de mantenimiento en las Pymes

\begin{tabular}{lc}
\hline Variable & Porcentaje (\%) \\
\hline $\begin{array}{l}\text { Política y objetivos } \\
\text { Identificación y clasificación } \\
\text { de equipos }\end{array}$ & 47 \\
$\begin{array}{l}\text { Análisis criticidad de equipos } \\
\text { Selección modelo } \\
\text { de mantenimiento }\end{array}$ & 64 \\
Análisis de modos de fallo & 50 \\
y efectos & \\
Planificación del & 48 \\
mantenimiento & 66 \\
Estimación tiempos & \\
de ejecución & \\
Programación del & 52 \\
mantenimiento & \\
Política de repuestos & 68 \\
Organización & 64 \\
Documentación & 48 \\
Presupuesto & 45 \\
Planificación (promedio) & $\mathbf{5 4}$ \\
\hline Fue: &
\end{tabular}

Fuente: Elaboración propia.

De igual forma no se ha definido formalmente una política de repuestos y la tendencia es mantener la mínima existencia de repuestos y solo para los equipos críticos; el personal de mantenimiento depende de la unidad de producción y la mayoría de empresas maneja órdenes de trabajo; están definidos los procedimientos para llevar a cabo el mantenimiento correctivo; existe previsión presupuestaria para la compra de repuestos, pago de nómina de mantenimiento y servicios contratados de mantenimiento (Tabla 2).

Al analizar estos resultados, se desprende que las empresas conciben que la gestión de mantenimiento está diri- 
gida más a reparar los equipos que se dañan que a mantener la planta en condiciones de operación y garantizar la capacidad de la misma para producir valor. Según Arata (2009:194), el concepto actual de gestión de mantenimiento tiene como prioridad prevenir fallas, de modo que se reduzcan las detenciones imprevistas en los equipos.

La confiabilidad de la gestión debe estar apoyada por un manejo adecuado de los materiales y repuestos, con políticas establecidas para controlarlos y administrarlos, además de personal entrenado en la función; las empresas deben integrarse a la cadena de abastecimiento y negociar convenios de suministro a través de los cuales los proveedores se comprometan a reabastecer en función de un nivel acordado, en lugar de ordenar los repuestos solo cuando se necesitan.

\subsection{Ejecución (hacer)}

La ejecución del plan de mantenimiento comprende únicamente actividades como lubricación y sustitución de piezas sometidas a desgaste lo que se corresponde con García (2003); el mayor porcentaje de ejecución de mantenimiento corresponde a reparación y corrección; se cuantifica el tiempo de producción perdido por averías (Tabla 3).

Al analizar como se ejecuta el mantenimiento, se deduce que al no existir técnicas de mantenimiento predictivo para anticipar y programar acciones críticas, no se garantiza la atención oportuna de las necesidades de mantenimiento; de esta manera, la asignación del tiempo y los recursos se decide en función de la urgencia de los problemas a atender.
Tabla 3

\section{Ejecución de mantenimiento} en las Pymes

\begin{tabular}{lc}
\hline Variable & Porcentaje (\%) \\
\hline Ejecución del plan & \\
de mantenimiento & 75 \\
Reparación y corrección & 78 \\
Ejecución (promedio) & 77 \\
\hline
\end{tabular}

Fuente: Elaboración propia.

\subsection{Verificar}

Con respecto a este factor, los resultados indican que se llevan registros de las averías pero no se realiza un análisis sistemático de las mismas para evitar su repetición; no se lleva control del tiempo empleado en las reparaciones; se manejan solo indicadores de disponibilidad; se mide informalmente la satisfacción de los clientes internos; no se lleva control de gastos de mantenimiento por equipo; se han introducido mejoras en las instalaciones así como cambios en los procedimientos destinados a aumentar la efectividad del mantenimiento (Tabla 4).

Para anticipar las necesidades de mantenimiento, se deben analizar las frecuencias de falla, analizar variaciones en las condiciones de operación que puedan conducir a fallas y desarrollar métodos de predicción de vida remanente de los equipos más críticos tomando en cuenta su régimen de utilización.

Más que asumir lo indicado por producción sobre la gestión de mantenimiento, es necesario hacer encuestas periódicas de satisfacción de los responsables de las áreas productivas atendidas, para asegurar la disponibilidad de los procesos más críticos para la producción, en lu- 
Gestión de mantenimiento en pymes industriales

Ortiz Useche, Alexis; Rodríguez Monroy, Carlos e Izquierdo, Henry

Tabla 4

Verificación de mantenimiento en las Pymes

\begin{tabular}{lc}
\hline Variable & Porcentaje (\%) \\
\hline Análisis de averías & 56 \\
Evaluación de la efectividad del mantenimiento & 57 \\
Medición satisfacción del cliente & 54 \\
Mejoras y/o modificaciones de instalaciones y procedimientos & 60 \\
Verificación (promedio) & $\mathbf{5 7}$ \\
\hline
\end{tabular}

Fuente: Elaboración propia.

gar de esperar la ocurrencia de las fallas, debe procurarse además, aumentar la robustez de las instalaciones mediante mejoras en su diseño y construcción.

\subsection{Actuar (mejora continua)}

Se detectaron esfuerzos dirigidos a mejorar la mantenibilidad; pero muy pocas empresas manejan el concepto de mantenimiento autónomo y los operarios de producción rechazan ocuparse de tareas simples de mantenimiento; se han impartido talleres sobre las $5 \mathrm{~S}$ pero las condiciones de orden y limpieza en general no son adecuadas (Tabla 5).

De acuerdo con González (2005: 116), si el personal de producción actúa de manera opuesta al concepto de mantenimiento autónomo y al orden y limpie-

Tabla 5

Mejora continua del mantenimiento en las Pymes

\begin{tabular}{lc}
\hline Variable & $\begin{array}{c}\text { Porcentaje } \\
(\%)\end{array}$ \\
\hline Mejora de la Mantenibilidad & 54 \\
Mantenimiento autónomo y 5 S & 56 \\
Mejora continua (promedio) & $\mathbf{5 5}$ \\
\hline
\end{tabular}

Fuente: Elaboración propia. za, significa que la dirección tampoco lo ha asumido como una iniciativa de mejora que defiende de forma inequívoca, por lo que es necesario pensar en un sistema de incentivos o reconocimiento, antes que imponer el concepto de manera obligatoria u abandonar el mismo.

En función de lo anterior se propone un modelo de gestión de mantenimiento para las pymes de Ciudad Guayana.

\section{Modelo de gestión de mantenimiento: Esquema de funcionamiento}

El modelo ha sido construido de acuerdo con los resultados obtenidos por la aplicación del análisis factorial exploratorio, que agrupó las veinte variables en términos de un menor número de variables conocidas como factores, presentándose finalmente cuatro factores representativos correspondientes al ciclo Planificar, Hacer, Verificar y Actuar (mejora continua).

El modelo se basa en el concepto de gestión por procesos y está orientado a incrementar la disponibilidad de los equipos; contiene las variables mínimas necesarias para el desempeño eficaz de 
la gestión de mantenimiento, cuya aplicación está al alcance de los recursos y capacidades de las pequeñas y medianas empresas.

La secuencia de funcionamiento del modelo presentado en el Diagrama 1, se basa en el modelo de Sistemas de Gestión ISO (2008:8) y en el modelo de gestión de mantenimiento propuesto por Parra y Crespo (2012:4).

En el esquema de funcionamiento del modelo, se ha representado al cliente tanto en la parte derecha como en la izquierda, para denotar que la gestión de mantenimiento comienza y acaba con el cliente, representado por la gestión productiva o subsistema de producción. A la izquierda, aparecen los requisitos del cliente: lo que el cliente quiere. Esto se transforma en un elemento de entrada para el sistema de gestión de mantenimiento (que se representa en el diagrama dentro de un rectángulo). Este elemento de entrada se introduce en el bloque superior Planificar.

El flujo principal del proceso que permite que se preste el servicio de mantenimiento al cliente, se muestra a lo largo

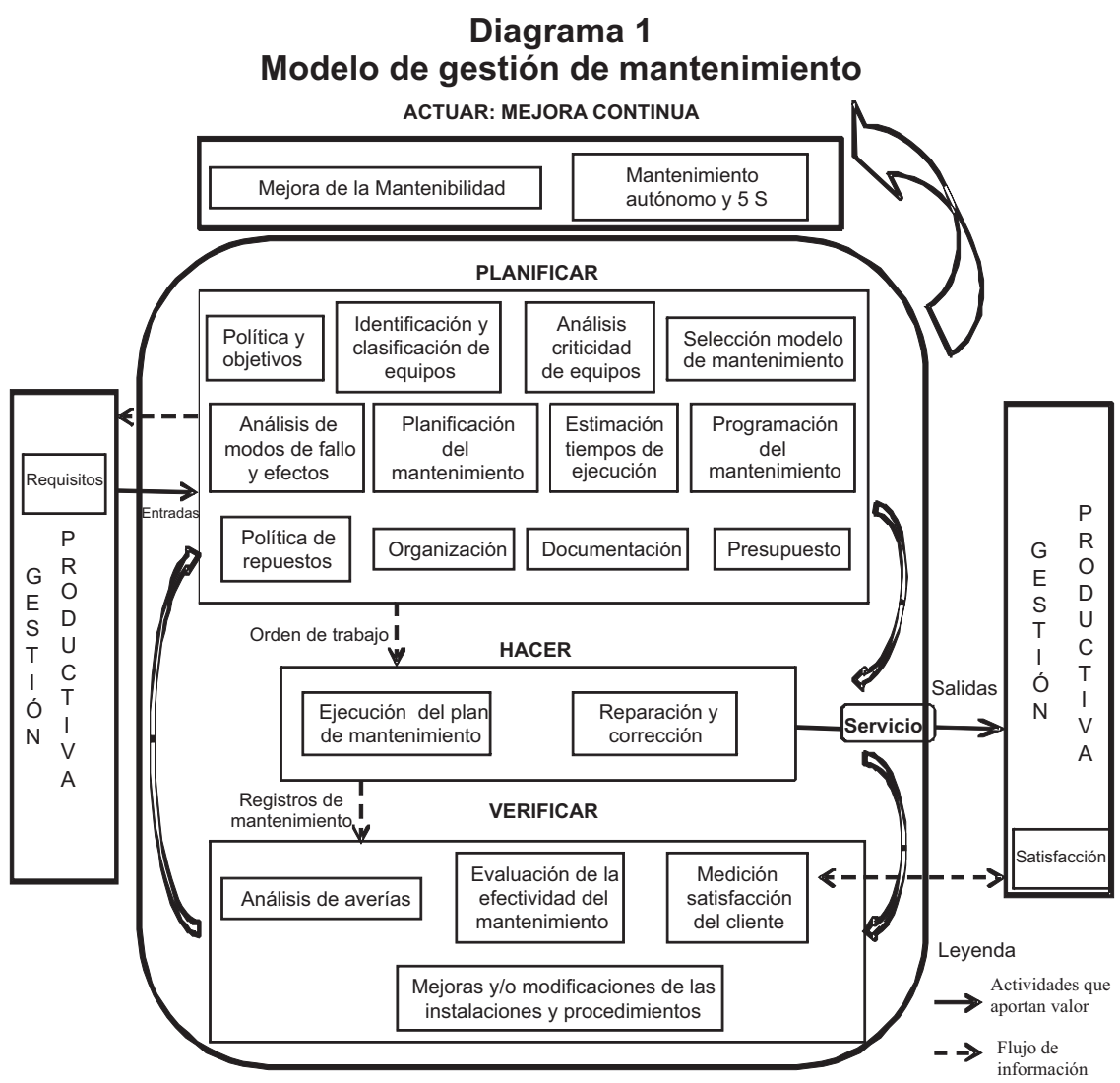

Fuente: Elaboración propia, basado en ISO (2008:8) y, Parra y Crespo (2012:4). 
de la parte central de la figura en el bloque Hacer.

El tercer bloque corresponde a las acciones Verificar, las cuales comprenden el seguimiento y medición de la gestión de mantenimiento contra los objetivos y planes y la toma de acciones correctivas y preventivas.

El bloque superior comprende las acciones correspondientes a actuar o mejora continua, dirigidas a mejorar continuamente la eficacia de la gestión de mantenimiento.

El modelo destaca la importancia de obtener información acerca de la satisfacción del subsistema de producción (la flecha discontinua de la derecha que apunta al bloque Verificar). Esto y otras mediciones y evaluaciones se convierten en información vital acerca del desempeño de la gestión de mantenimiento.

El modelo muestra también la necesidad de mantener comunicación con los clientes, de forma que se conozca lo que esperan y que sea capaz de satisfacerlos (la flecha discontinua de la izquierda).

Es importante que las pymes industriales analizadas internalicen el modelo propuesto en aras de mejorar su gestión de mantenimiento y con esto incrementar la eficiencia en sus operaciones productivas.

\section{Conclusiones}

El modelo de gestión de mantenimiento para las pymes industriales presentado como producto de esta investigación, está conformado por cuatro factores, que agrupan veinte variables, resultado de la aplicación del análisis factorial exploratorio. Los factores extraí- dos se catalogaron en las áreas correspondientes a Planificación de Mantenimiento (Planificar), Ejecución del Mantenimiento (Hacer), Medición, Evaluación y Análisis (Verificar) y Actuar (Mejora Continua).

Como resultado del análisis de la gestión de mantenimiento, el conjunto de empresas evaluadas obtuvo una calificación de $57 \%$ de cumplimiento, lo cual pone de relieve la debilidad del sistema de gestión de mantenimiento en las pymes industriales del Estado Bolívar, particularmente en las áreas: planificar y mejora continua.

En las etapas de evolución de la gestión del mantenimiento, la mayor parte de las pymes evaluadas se encuentran en la etapa inicial (mantenimiento correctivo), siendo su norma de actuación la respuesta solamente a la ocurrencia de averías; muy pocas apoyan su gestión en la planificación y el control.

Solo la mitad de las empresas poseen personal de mantenimiento calificado; en el resto, el personal asignado no posee las competencias requeridas y no existen programas de capacitación y formación, lo que afecta la eficacia de la gestión.

En general, la gestión de mantenimiento no es vista como un factor para mejorar la competitividad de la empresa, por lo que el reto planteado a las pymes de la región es evolucionar del paradigma de la corrección a la práctica de la prevención; no obstante, para asumir este reto, el empresario debe concebir al mantenimiento como un elemento clave de la competitividad en lugar de un mal necesario, ya que el mismo, al servir de soporte a la gestión de la producción tiene un efecto en la reducción de los costos. 


\section{Referencias bibliográficas}

AENOR (2007). Guía para la preparación de contratos de mantenimiento. UNEEN 13269. Asociación Española de Normalización y Certificación, pp 8.

Amendola, Luis (2006). Gestión de proyectos de activos industriales. Editorial Universidad Politécnica de Valencia. Valencia, España.

Arata, Adolfo (2009). Ingeniería y gestión de la confiabilidad operacional en plantas industriales. Ril Editores. Santiago, Chile, pp. 131, 134, 194.

Asamblea Nacional (2012). Ley Orgánica del Trabajo, las Trabajadoras y los Trabajadores. Gaceta Oficial de la República Bolivariana de Venezuela $\mathrm{N}^{\circ}$ 6.076 Extraordinario, Venezuela.

Cabello, Juan (2002). Propuesta doctrinal para la elección de un programa de mantenimiento. Tesis doctoral. Departamento de Economía y Administración de Empresas. Universidad de Málaga. Málaga, España, pp. 117.

Campbell, John; Jardine, Andrew y McGlynn, Joel. (2010). Asset Management Excelence: Optimizing Equipment Life-Cycle Decisions. CRC Press, pp. 219.

COVENIN (1993). Manual para evaluar los sistemas de mantenimiento en la industria. Comisión Venezolana de Normas Industriales, 2500-93, pp 4.

Creus Sole, Antonio (2005). Fiabilidad y seguridad, su aplicación en procesos industriales. Marcombo, S.A., Cataluña, España, pp. 95.

Cuatrecasas, Lluis; Torrell Francesca (2010). TPM en un entorno Lean Management. Profit Editorial I., S. L. Barcelona, España, 36.

García, Santiago (2003). Organización y gestión integral de mantenimiento. Ediciones Díaz de Santos. Madrid, Espa- ña, pp. 7-14, 25, 43, 46, 79, 103, 111, 250.

Gil, María; Giner, Fernando (2010). Como crear y hacer funcionar a una empresa. ESIC Editorial, Madrid, España, pp. 199-203.

Goble, William (2010). Control Systems Safety Evaluation and Reliability. International Society of Automation. North Carolina, USA, pp. 87.

González, Francisco (2004). Auditoría del mantenimiento e indicadores de gestión. Fundación Confemetal. Madrid, España, pp. 39, 393-394.

González, Francisco (2005). Teoría y práctica del mantenimiento industrial avanzado. Fundación Confemetal. Madrid, España, pp. 109,116.

ISO (2008). Sistemas de gestión de la calidad. Requisitos. 9001:2008. Secretaría Central de ISO. Ginebra, Suiza, pp. 8.

Kister, Timothy; Hawkins, Bruce (2006). Maintenence planning and scheduling: streamline your organization for a lean environment. Elsevier Butterworth-Heinemann, USA.

Martínez, Isabel; Santero, Rosa; Sánchez, Luis; Marcos, Miguel (2009). Factores de competitividad de la Pyme española. Fundación EOI, Madrid, pp. 28,94.

Oliva, Karim; Arellano, Madelein; López, María; Soler, Karen (2010). Sistemas de información para la gestión de mantenimiento en la gran industria del estado Zulia. Revista Venezolana de Gerencia, Vol. 15, Núm. 49, Venezuela, Centro de Estudios de la Empresa, Universidad del Zulia, pp. 125-140.

Parra, Carlos y Crespo, Adolfo (2012). Ingeniería de Fiabilidad y Mantenimiento aplicada en la Gestión de Activos. Desarrollo y aplicación práctica de un Modelo de Gestión del 
Gestión de mantenimiento en pymes industriales

Ortiz Useche, Alexis; Rodríguez Monroy, Carlos e Izquierdo, Henry

Mantenimiento (MGM). INGEMAN, España, pp. 4.

Plaza, Alejandro (2009). Apuntes teóricos y ejercicios de aplicación de gestión del mantenimiento industrial. Editor: Alejandro Plaza Tovar. Madrid, pp. 132.

Salvador, Manuel y Gargallo, Pilar (2006). Análisis Factorial. Universidad de Zaragoza. España. http://www.5campus.com/leccion/factorial. Consulta Mayo 2011.

Sistema Económico Latinoamericano y del Caribe, SELA (2009). Políticas e instrumentos para enfrentar el impacto de la crisis económica internacional sobre las pequeñas y medianas empresas (PYMES) de América Latina y el Caribe. Secretaría Permanente del Sela. Caracas. Venezuela. Mayo SP/Di Nº6-09.
Stephens, Matthew (2010). Productivity and Reliability-Based Maintenance Management. Purdue University Press, USA, pp. 7,30.

Wenyuan, Li (2005). Risk Assessment of Power Systems: Models, Methods, and Applications. Wiley-IEEE Press, USA.

Zevallos, Emilio (2008). Pymes, tecnología y gestión de información; artículo del libro "Pymes: Visión Estratégica para el Desarrollo Económico y Social", publicación del Sistema Económico Latinoamericano y del Caribe (SELA), pp. 32-33.

Zylberberg, Ariel (2006). Introducción al análisis racional de repuestos. En www.mantenimientoplanificado.com/ Artículos/GESTIONMANTENIMIENTOARTICULOS/Introducción de los repuestos basado en riesgos/. Consulta Mayo 2011. 